\title{
SOME REMARKS ON THE MULTIPLICATIVE GROUP OF A SFIELD
}

\author{
EUGENE SCHENKMAN ${ }^{1}$
}

Introduction. In this note when $K$ is a sfield then $K^{\prime}$ will denote the multiplicative group of $K$. We shall show that if $H$ is any subfield of $K$ or a noncommutative subsfield of the sfield $K$ (with some few exceptions) and if $H^{\prime}$ is subinvariant in $K^{\prime}$ then $H^{\prime}$ is invariant in $K^{\prime}$ and hence $H$ is either $K$ itself or in the center of $K$. This result extends the Cartan-Brauer-Hua theorem (cf. [1]).

Notation. If $M$ is a subset of $K$ then $Z(M)$ will denote the centralizer of $M$; this is a sfield, its multiplicative group will be denoted by $Z^{\prime}(M)$. The normalizer of $M$ in $K^{\prime}$ will be denoted by $N(M)$; and the normalizer of $N(M)$ by $N^{2}(M)$. If $J$ is an invariant subgroup of $L$ we shall write $J \Delta L$. If $J$ is subinvariant in $L$, that is, if $J$ is a member of a composition series of $L$ we shall write $J \Delta \Delta L$.

Conclusions. The first lemma is essentially the argument of [1].

LEMMA 1. If $M$ is a subsfield of the sfield $K$ and if $x$ is in $N(M)$ but not in $M$ nor in $Z(M)$ then for all $m$ in $Z(M) \cap M^{\prime}, m+x$ is not in $N(M)$.

Proof. Since $x$ is not in $Z(M)$ there exists an $n$ in $M$ such that $n x=x n^{\prime}$ where $n^{\prime}$ is in $M$ and $n^{\prime} \neq n$. Then $(m+x)^{-1} n(m+x)$ $=(m+x)^{-1}\left[n m+x n^{\prime}+m n^{\prime}-m n^{\prime}\right]=(m+x)^{-1}\left(n m-m n^{\prime}\right)+n^{\prime}$. If the left member were in $M$ then so would be the right member and consequently $(m+x)^{-1}\left(n m-m n^{\prime}\right)$; then $(m+x)^{-1}$ and hence $x$ are in $M$ contrary to hypothesis.

Corollary 1 (Cartan-Brauer-Hua Theorem). The only invariant subsfields of $K$ are $K$ itself and subfields of the center of $K$.

Proof. Suppose $M$ is an invariant subsfield of $K$ not equal to $K$ nor contained in the center of $K$. Then there is a nonzero $x$ not in $M^{\prime}$ and a nonzero $y$ not in $Z(M)$; and one of the three elements $x, y$, and $x y$ is in neither $M^{\prime}$ nor in $Z^{\prime}(M)$. For if two of these elements were in one of these sfields it would follow that the third is also there. It follows from the lemma that there is a nonzero element outside $N(M)$ contrary to hypothesis.

Received by the editors August 19, 1957.

1 The author is indebted to the National Science Foundation for support. 
Lemma 2. If II and $M$ are subsfields of $K, H$ not contained in $M$, and if $I I^{\prime}$ is contained in $N(M)$, then $H$ is contained in $Z(M)$.

Proof. If $H$ were not in $Z(M)$, then there would be an $x$ in $H$, $x$ not in $Z(M)$. Since $H$ is not in $M$ there is a $y$ in $H^{\prime}, y$ not in $M$. Now the three elements $x, y$, and $x y$ are all in $H^{\prime}$ and hence in $N(M)$. One of them is in neither $M$ nor in $Z(M)$ for if any two are in one of these sfields then the third is there also. This is a contradiction of Lemma 1 since the elements $x+1, y+1$, and $x y+1$ are also in $H^{\prime}$ and hence in $N(M)$. We conclude that $H$ is contained in $Z(M)$ as the lemma asserts.

CoRollary 2. If $M$ is a sfield which is the centralizer of its center $H$ then $N(M)=N^{2}(M)=N(H)=N^{2}(H)$.

Proof. $H$ is invariant in $N(M)$ and hence $N(M)$ is contained in $N(H)$. On the other hand $M$ is contained in $N(H)$ and is normal in $N(H)$ since $M$ is the centralizer of $H$. Hence $N(H)$ is contained in $N(M)$ and therefore $N(H)=N(M)$.

Now suppose there is a $y$ in $N^{2}(M), y$ not in $N(M)$. Then $y$ transforms $H$ into a conjugate field $G$ not contained in $H$, but contained in $N(M)$ and invariant in $N(M)$. By Lemma $2, G$ is contained in $Z(H)$ $=M$ and, since $M$ is in $N(M), G$ is invariant in $M$. But then by the Cartan-Brauer-Hua theorem since $G$ is not contained in $H, G$ must be equal to $M$. It follows that $M$ is Abelian and hence equal to $H$ equal to $G$ contrary to the fact that $y$ was chosen out of $N(M)$. We conclude that $N(M)=N^{2}(M)$.

REMARK. If $M$ is a maximal subfield of the sfield $K$ then $N(M)$ $=N^{2}(M)$. For by the maximality $M$ is the centralizer of its center.

THEOREM 1. If $F$ is a proper subfield of the sfield $K$ and if $F^{\prime}$ is subinvariant in $K^{\prime}$ then $F$ is in the center of $K$.

Proof. Suppose $F$ is not in the center of $K$ and suppose that $F^{\prime} \triangle G_{1} \triangle G_{2} \triangle \cdots \triangle G_{n}=K^{\prime}$. We shall show that the sfield $\bar{F}$ generated by all the conjugates of $F^{\prime}$ in $K^{\prime}$ is Abelian. This will give a contradiction to the Cartan-Brauer-Hua theorem since then $\bar{F}^{\prime}$ is invariant in $K^{\prime}$ but not equal to $K^{\prime}$ nor in the center of $K^{\prime}$.

$\bar{F}$ is not in the center of $K$ since $F$ is not, and $\bar{F}$ contains $F . \bar{F}$ is not equal to $K$ since that would imply $K$ is Abelian and $F$ would be in the center of $K . \bar{F}$ is invariant in $K$ since it is the sfield generated by an invariant subset of $K^{\prime}$. Thus the theorem is proved when we show that $\bar{F}$ is Abelian. This will be done by induction on the length $n$ of the composition series containing $F^{\prime}$.

Suppose then that for $j$ in some set $J, F_{j}^{\prime}$ are all the conjugates of 
$F^{\prime}$ by elements of $G_{2}$. Then each $F_{j}^{\prime}$ is normal in $G_{1}$ and hence by Lemma 2 each $F_{j}^{\prime}$ is in the centralizer of all the others. It follows that the sfield $F_{1}$ generated by the $F_{j}$ is a field. Suppose now that we have shown that the sfield $F_{m}$ generated by all the conjugates of $F^{\prime}$ in $G_{m}$ is a field. It is easy to check that $F_{m}^{\prime}$ is normal in the group generated by $F_{m}^{\prime}$ and $G_{m+1}$. If $F^{* \prime}$ is a conjugate of $F^{\prime}$ in $G_{m+1}$ then again by Lemma $2 F^{*}$ is in the centralizer of $F_{m}$ and hence in particular $F$ and $F^{*}$ commute elementwise; by a symmetry argument all the conjugates of $F^{\prime}$ contained in $G_{m+1}$ commute elementwise and hence the sfield $F_{m+1}$ that they generate is a field. Then by induction we see that $F_{n}=\bar{F}$ is Abelian as was to be shown. This proves the theorem.

LemMa 3. If $M$ is a noncommutative subsfield of $K$ and if $N(M)$ $\neq N^{2}(M)$ then $N(M)$ is of index 2 in $N^{2}(M)$ and $Z^{\prime}(M)$ is the only other conjugate of $M^{\prime}$ contained in $N(M)$. Furthermore $N^{2}(M)$ is its own normalizer in $K^{\prime}$ and $N^{2}(M) \neq K^{\prime}$ provided that the center of $M$ contains at least 5 elements of the center of $K$.

Proof. Suppose $N(M) \neq N^{2}(M)$ and that $N(M)$ is of index $m>2$ in $N^{2}(M)$. Then there are at least three conjugates $M^{\prime}, M^{* \prime}$ and $M^{* * \prime}$ contained in $N(M)$ and having $N(M)$ for normalizer. It follows from Lemma 2 that any two of these are in the centralizer of the third and since $N(M)$ is the normalizer of each, the sfield generated by each pair is in $N(M)$. Now since $M$ is not commutative there is an $x$ in $M, x$ not in $Z(M)$. Since $M$ and $M^{*}$ are distinct conjugates there is a $y$ in $M^{*}$, not in $M$. Then $x+y$ is not in $M$ nor in $Z(M)$. But this contradicts Lemma 1 since both $x+y$ and $x+y+1$ are in $N(M)$ since they are in the centralizer of $M^{*}$. We conclude that if $N(M) \neq N^{2}(M)$ then $N(M)$ is of index 2 in $N^{2}(M)$.

Now when $N(M)$ is of index 2 in $N^{2}(M)$ then there is at least one conjugate $M^{* \prime}$ of $M^{\prime}$ in $N(M) . M^{*}$ is contained in $Z(M)$ and in fact is equal to $Z(M)$; for if $M^{*}$ were properly contained in $Z(M)$ then by symmetry $M$ would be properly contained in a sfield $H$ such that $H^{\prime}$ is in $N(M)$. But then by Lemma $2, H$ would be contained in $Z(M)$, whence $M$ would be also and hence $M$ would be Abelian contrary to hypothesis. We conclude that $M^{*}$ must be equal to $Z(M)$.

Now if $M^{* * \prime}$ were another conjugate of $M^{\prime}$ in $N(M)$ then $M^{* *}$ would be contained in $Z(M)=M^{*}$ which contradicts the fact that one conjugate cannot be contained in another. We conclude that there are only two conjugates of $M^{\prime}$ in $N(M)$ when the index of $N(M)$ in $N^{2}(M)$ is 2 as the lemma asserts.

The following Lemma is now needed to finish the proof of Lemma 3. 
Lemma 4. There are no noncommutative subsfields $L$ and $M$ of $K$ such that $L \cap N(M)$ is of index 2 in $L^{\prime}$ provided that the center of $M$ contains at least 5 elements of the center of $K$.

Proof of Lemma 4. Let $L^{*}$ denote $L \cap N(M)$. We shall show first that every $x$ in $L^{*}$ is either in $M$ or in $Z(M)$. For suppose there is an $x$ in $L^{*}$ but not in $M$ nor in $Z(M)$. Then by Lemma $1, x+1$ and $x-1$ are not in $L^{*}$ and since the index of $L^{*}$ in $L^{\prime}$ is 2 it follows that $(x+1)(x-1)=x^{2}-1$ must be in $L^{*}$ as is also $x^{2}$. It follows again from Lemma 1 that $x^{2}$ must be in $M$ or in $Z(M)$.

Now if the characteristic of the sfield is not 2 or 3 , let $a=1, b=3$, $c=2$, and $d=-1$. If the characteristic is 3 let $a=c=1$ and let $b$ and $d$ be distinct elements of $M$ in the center of $K$ but not 0,1 , or 2 . If the characteristic is 2 let $a, b, c, d$ be elements of $M$ in the center of $K$ but not 0 or 1 and such that $a+b \neq 0, a+b+1 \neq 0$ and $a=c$, $d=b+1$. Then none of the elements $x+a, x+b, x+c, x+d, x+a+1$, $x+b-1, x+c+1, x+d-1$ is in $L^{*}$ so that $(x+a)(x+b)=x^{2}+(a+b) x$ $+a b$ is in $L^{*}$ as is also $(x+a+1)(x+b-1)=x^{2}+(a+b) x+(a+1)$ $\cdot(b-1)$. It follows again from Lemma 1 that $x^{2}+(a+b) x+a b$ and hence $x^{2}+(a+b) x$ is in $M$ or in $Z(M)$. Similarly by using $c$ in place of $a, d$ in place of $b$ we see that $x^{2}+(c+d) x$ is in $M$ or in $Z(M)$. But then two of the three elements $x^{2}, x^{2}+(a+b) x, x^{2}+(c+d) x$ are in the same sfield $M$ or $Z(M)$ and by subtraction of one from the other we see that $x$ is also there contrary to the supposition that $x$ was neither in $M$ nor in $Z(M)$. We conclude that every element of $L^{*}$ is in $M$ or in $Z(M)$.

Now if $L^{*}$ were in $M$ or if $L^{*}$ were in $Z(M)$ then that sfield contains all the squares of elements of $L$ since $L^{*}$ is of index 2 in $L^{\prime}$ and hence contains $L$ itself since by Theorem 5 of [2] the square elements of a noncommutative sfield generate the whole sfield. This, of course, means that $L$ is contained in $M$ and hence $N(M)$ contrary to the fact that $L \cap N(M)$ is of index 2 in $L$.

On the other hand, if there are elements $x$ and $y$ of $L^{*}, x$ in $M$ but not in $Z(M)$ and $y$ in $Z(M)$ but not in $M$ then $x y$ is in $L^{*}$ but in neither $M$ nor $Z(M)$ contrary to what was shown above. This proves Lemma 4.

We now continue the proof of Lemma 3. If $N(M) \neq N^{2}(M)$ and if $N^{2}(M)$ is not its own normalizer then there is a conjugate $N^{*}$ of $N(M)$ also of index 2 in $N^{2}(M)$ and in $N^{*}$ a conjugate $M^{* * \prime}$ of $M^{\prime}$ such that either $M^{* * \prime} \cap N(M)$ is of index 1 or 2 in $M^{* * \prime}$. We rule out the possibility of this index being 2 because of Lemma 4 , while if the index is 1 then $M^{* * \prime}$ is contained in $N(M)$ and there are three distinct 
conjugates of $M^{\prime}$ in $N(M)$ contrary to the first statement of the lemma already proved. This concludes the proof of Lemma 3.

THEOREM 2. If $M$ is a proper noncommutative subsfield of a sfield $K$ containing at least 5 elements of the center of $K$, then $M^{\prime}$ is not subinvariant in $K^{\prime}$.

Proof. Suppose $M^{\prime} \Delta G_{1} \Delta G_{2} \Delta \cdots \Delta G_{n}=K^{\prime}$ and suppose $r$ is the largest integer so that $G_{r}$ is contained in $N^{2}(M)$. Then $r \neq n$ since $N^{2}(M) \neq K^{\prime}$ by Lemma 3. Now if $y$ is any element of $G_{r+1}$ then $y$ transforms $M^{\prime}$ into a conjugate $M^{* \prime}$ contained in $G_{r}$ and hence in $N^{2}(M)$. It follows from Lemmas 3 and 4 that $M^{*}$ is either $M$ or $Z(M)$ and hence $y$ is in $N^{2}(M)$; consequently $G_{r+1}$ is also in $N^{2}(M)$ contrary to the choice of $r$. We conclude that $M^{\prime}$ cannot be subinvariant in $K^{\prime}$.

\section{BIBLIOGRAPHY}

1. Richard Brauer, On a theorem of H. Cartan, Bull. Amer. Math. Soc. vol. 55 (1949) pp. 619-620.

2. L. K. Hua, Some properties of sfields. Proc. Nat. Acad. Sci. vol. 35 (1949) pp. 533-537.

Louisiana State University 